preceded the development of erythema in our cases, representing a harbinger for the more severe grade of rejection that eventually developed. Our experience was consistent with other VCAs in that donor specific antibodies did not develop, despite a severe Banff Grade. Consistent use of topical calcineurin inhibitor based immunosuppression on the allograft skin may be helpful in warding off future episodes, as our patient has been rejection free now for 18 months. To date, no histologic signs of chronic rejection were present on 2-year protocol surveillance biopsy. We have added rapamycin to the current drug regimen, with concurrent reduction of tacrolimus dosing for renal protection, which has been demonstrated in cardiac transplantation to deter the intimal hyperplasia/vasculopathy associated with chronic rejection.

Metagenomic characterization of influenza virus: bacteria super-infections associated with death and survival

Josh Klonoski, MD PhD ${ }^{1}$, Matthew Williams and Victor Huber ${ }^{1}$ The University of Utah School of Medicine

OBJECTIVES/SPECIFIC AIMS: To better understand host, viral and bacterial responses underlying disparate outcome. METHODS/ STUDY POPULATION: We utilized metagenomic analysis of 559 genes, the NanoString nCounter Immunology Panel-Plus kit and FFPE mouse lungs from a published BSI time course. RESULTS/ ANTICIPATED RESULTS: Results show an overall increased level of gene expression during BSIs associated with survival when compared to gene expression during peak viral titers. Early viral clearance and the presence of S. pyogenes in the lungs of TX98 infected lungs 24 hours after BSI was confirmed. Host responses tied to differences in early viral detection and clearance consisted of RIG-I, OAS, TLR3, TLR8 and TLR9. Key changes were noted in the expression of type I and II interferons, complement proteins, cytokines, chemokines, Fc receptors, scavenger receptors, the immunoproteasome and genes associated with T, B, Treg and NK cell activation. Interestingly, there was no significant increase in host antimicrobial peptides during BSIs associated with survival while CAMP and Nos2 were significantly increased $24 \mathrm{hrs}$ prior to death in lethal BSIs. DISCUSSION/ SIGNIFICANCE OF IMPACT: To our knowledge this is the first side by side metagenomics study of influenza BSIs associated with death and survival. Results offer mechanistic insight into clinical outcomes.

3373

\section{Modulation of Hedgehog Signaling Alters Immune Infiltration in Pancreatic Cancer}

Nina Steele ${ }^{1}$, Valerie Irizarry-Negron, Veerin Sirihorachai, Samantha Kemp, Eileen Carpenter, Christopher Halbrook, Costas Lyssiotis, Filip Bednar, Timothy Frankel, Benjamin Allen and Marina Pasca di Magliano

${ }^{1}$ University of Michigan School of Medicine

OBJECTIVES/SPECIFIC AIMS: Pancreatic ductal adenocarcinoma (PDA) has a dismal 5-year survival rate of 9\%, making this disease one of the deadliest human malignancies (https://seer.cancer.gov/). Primary barriers to the treatment of pancreatic cancer include extensive stromal interactions and sustained immune suppression. Aberrant Hedgehog $(\mathrm{HH})$ pathway activity is a hallmark of pancreatic tumorigenesis. Tumor-derived $\mathrm{HH}$ ligands signal in a paracrine fashion to the surrounding stroma to influence tumor growth. Expression of $\mathrm{HH}$ ligands increases during PDA progression, and previous work has shown that genetic deletion of Sonic HH (Shh) from the epithelium of mice with pancreatic tumors results in increased Indian $\mathrm{HH}$ (Ihh) expression. This research aims to investigate the translational impact of changes in immune infiltration following deletion of IHH in a preclinical mouse model of pancreatic cancer. METHODS/STUDY POPULATION: Ihh was deleted in tumor cells lines (IhhKO) derived from a genetically engineered mouse model of pancreatic cancer (LSL-KrasG12D/+;LSL-TrpR270H;P48-Cre), using CRISPR/Cas-9 gene editing to assess the role of Ihh in the tumor microenvironment. The level of $\mathrm{HH}$ signaling was determined using tumor cell co-cultures with GlillacZ fibroblasts (derived from mice with a lacZ reporter allele knocked into the Gli1 locus), in which Beta Galactosidase activity serves as a readout for $\mathrm{HH}$ signaling. WT and IhhKO tumor cells were orthotopically transplanted into the pancreas of syngeneic C57BL/6 mice. Human pancreas samples were obtained from surgical resection of pancreatic adenocarcinoma, or fine needle biopsy procedure (FNB). Immune profiling of mouse and human pancreatic tumors was performed using Cytometry Timeof-Flight analysis (CyTOF), and tumor composition was analyzed by single-cell RNA sequencing (scRNA seq). In vitro cultures with pancreatic fibroblasts treated with either WT or IhhKO tumor cell conditioned media (CM) were cultured with bone-marrow derived macrophages to assess tumor crosstalk. RESULTS/ANTICIPATED RESULTS: Tumor cells lacking Ihh were generated through CRISPR/ Cas- 9 deletion, and this was confirmed by qRT-PCR. Co-culture of IhhKO tumor cells with GlillacZ fibroblasts results in decreased Gli1 expression both in vitro and in vivo. Immune profiling revealed that tumors lacking Ihh have significantly fewer tumor associated macrophages $(\mathrm{CD} 11 \mathrm{~b}+/ \mathrm{F} 4 / 80+/ \mathrm{CD} 206+)$, resulting in decreased presence of immunosuppressive factors such as arginase 1 and PDL1. Immune phenotyping of human pancreatic tissues revealed similar populations of immunosuppressive myeloid cells present in tumors. In vitro co-cultures demonstrated that, in the presence of bonemarrow derived macrophages, immunosuppressive IL- 6 production was reduced in pancreatic fibroblasts cultured with IhhKO-CM, as compared to fibroblasts cultured with WT-CM, providing mechanistic insight into the in vivo phenotype observed. Further, scRNA seq analysis suggests that modulation of $\mathrm{HH}$ signaling in the tumor microenvironment alters chemokine and immunomodulatory signaling pathways driven by fibroblasts in the pancreatic tumor microenvironment. DISCUSSION/SIGNIFICANCE OF IMPACT: HH signaling in pancreatic fibroblasts contributes to the establishment of an immune suppressive environment in pancreatic cancer. Combining methods to target $\mathrm{HH}$ signaling and immune checkpoint therapy has translational potential in treating pancreatic cancer patients.

\section{4}

\section{Naltrexone as a Diagnostic Tool in Ocular Neuropathic Pain}

Nicholas Fowler Dr. ${ }^{1}$, Romulo Albuquerque ${ }^{2}$, Jooyoung Cho ${ }^{2}$, Nicholas Bell ${ }^{2}$, Paras Vora ${ }^{2}$ and Greg Botzet ${ }^{2}$

${ }^{1}$ University of Kentucky Center for Clinical and Translational Science and ${ }^{2}$ University of Kentucky College of Medicine

OBJECTIVES/SPECIFIC AIMS: The study aims to track and correlate ocular neuropathic symptoms, corneal sensitivity and dry-eye like pain, after scleral buckle and posterior vitrectomy surgeries. The goal is to identify a population of patients that receive these retinal surgeries that experience ocular neuropathic pain. METHODS/ STUDY POPULATION: Methods - Prospective and Retrospective cohort studies were designed with the follow cohorts: scleral buckle, posterior vitrectomy, and control. Typical follow up for SB/PV 